Erschienen in: Harras, Gisela (Hrsg.): Die Ordnung der Wörter. Kognitive und lexikalische Strukturen. - Berlin, New York: de Gruyter, 1995. S. 4-29. (Institut für deutsche Sprache. Jahrbuch 1993)

PETER ROLF LUTZEIER

\title{
Lexikalische Felder - was sie waren, was sie sind und was sie sein könnten
}

\section{Zur Einstimmung}

Lexikalische Felder, Wortfelder, Wortfeldtheorie: Gehört ja! Trier usw. ... Aber Näheres nie so recht erfahren. Diese Reaktion könnte nicht nur auf Sie zutreffen; ich vermute, sie würde sogar auf die Mehrzahl aller Linguisten/Linguistinnen zutreffen. Nicht sehr viel besser kommen Wortfelder dann auch in Einführungen zur Linguistik oder spezieller zur Semantik weg: Erwähnt werden sie mit ein, zwei Beispielen sehr wohl, aber damit hat es sich auch schon - man hat den Eindruck, einer lästigen Pflicht wird Genüge getan, ohne daß man sich für das Gesamte viel davon verspricht. Immerhin, die fast obligatorische Erwähnung könnte ja auch auf etwas Positives an der Sache hinweisen, nämlich auf die Überzeugung, daß der Begriff 'Wortfeld' eigentlich intuitiv über ein, zwei Beispiele relativ leicht zu vermitteln sei. Nehmen wir die Verben der Bewegung: gehen, schreiten, schleichen, rennen usw. oder die Adjektive für Angstgefühle: ängstlich, bange, furchtsam, panisch usw. - solche Gruppen von Wörtern bilden Wortfelder. Damit kann man sich doch ein Bild von der Sache machen, oder etwa nicht? Aber weshalb geht es meistens über diesen Stand nicht hinaus? Man glaubt, diese eingängige, offenbar tief in uns verankerte Vorstellung von Gruppierungen von Wörtern brauche und vielleicht könne auch gar nicht weiter konkretisiert werden. Die intuitive Stärke der Konzeption wäre also ihre beschreibungstechnische Schwäche zugleich. Letzteres wird nun im Verlauf meiner Ausführungen zu relativieren sein. Einige der häufig angenommenen Schwachstellen haben eventuell eher mit der zu beschreibenden Sache - also der Sprache - als mit dem beschreibenden Instrument - also der Konzeption 'Wortfeld' - zu tun. Mit anderen Worten, man dürfte dann überhaupt nicht mehr von Schwachstellen der Wortfeldtheorie reden, sondern müßte vielmehr von Fakten einer natürlichen Sprache, insbesondere im Bereich der Kategorisierung, reden.

\section{Zum Umfeld der Konzeption}

Wortfelder, lexikalische Felder, darin sind wir uns wohl alle einig, sollen einen Beitrag zur Erfassung der lexikalischen Bedeutung leisten. Obwohl wir die lexikalische Bedeutung als eine holistische Entität ansehen müssen, sollte ihre Beschreibung mindestens vier Dimensionen vorse- 
hen. ${ }^{1}$ Zwei davon sehe ich als interne Dimensionen an - Dimensionen, die das einzelne Wort betreffen - und zwei davon sehe ich als externe Dimensionen an - Dimensionen, die das einzelne Wort in Beziehung zu anderen Wörtern setzen. Vgl. Sie für das folgende das Diagramm 1:

Dimensionen für die Beschreibung der lexikalischen Bedeutung

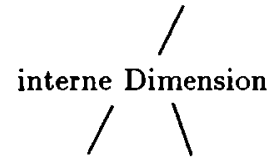

Dimension der Dimension des

Polysemie

\begin{tabular}{|l|}
\hline Lesarten ein \\
und desselben \\
Wortes \\
\end{tabular}

internen Aufbaus

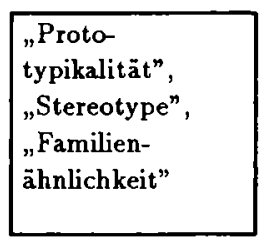

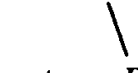

externe Dimension

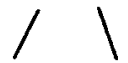

paradigmatische syntagmatische

Dimension

\begin{tabular}{|l|}
\hline Lesarten \\
verschiedener \\
Wörter \\
"Wortfeld- \\
theorie”, \\
„Sinnrelationen"
\end{tabular}

Dimension

"Kollokation",
"wesenhafte
Bedeutungs-
beziehungen".
"Assoziation"

Beginnen wir mit den internen Dimensionen: Beim Sprechen oder Schreiben ist es uns nicht bewußt, aber ein Moment des Nachdenkens oder ein Blick in ein vernünftiges Wörterbuch öffnet uns die Augen über die Tatsache, daß die meisten Wörter mehrdeutig sind. Bei Mehrdeutigkeit eines Wortes reicht eine Bedeutungsangabe nicht mehr aus, es müssen mehrere Lesarten voneinander unterschieden werden. Somit haben wir erstens die Dimension der Polysemie, die sich mit Kontrasten und $\mathrm{Zu}-$ sammenhängen der Lesarten ein und desselben Wortes beschäftigt. Nun ist aber jede einzelne Lesart auch in sich selbst strukturiert, wie uns wichtige psychologische und philosophische Einsichten aus teilweise neuerer Zeit nahegebracht haben. Deshalb ergibt sich zweitens die Dimension des internen Aufbaus einzelner Lesarten, bei der insbesondere Prototypikalitätseffekte, Stereotype und Familienähnlichkeiten zu berücksichtigen sind. Dank wichtiger klassischer Einsichten der Linguistik wissen wir, daß unsere Vorstellungen über einzelne Lesarten von Wörtern geschärft werden, wenn wir uns um den Platz des Wortes im Wortschatz kümmern; d.h. also die Aufgabe der Lexikologie ernst nehmen. Damit kommen wir zu den externen Dimensionen: Niemand kann den gesamten Wortschatz

1 Für mehr Informationen zu meiner Bedeutungskonzeption vgl. Lutzeier (1985a) und (1993c). 
einer natürlichen Sprache auf einmal behandeln, weshalb wir uns sinnvollerweise auf Teile des Wortschatzes konzentrieren. Falls wir uns dabei auf Wörter mehr oder weniger einer Wortart in der 'oder'-Beziehung beschränken, haben wir es mit der paradigmatischen Dimension zu tun. Diese Dimension beschäftigt sich mit Kontrasten und Zusammenhängen der Lesarten verschiedener Wörter. Insofern darf diese paradigmatische Dimension nicht mit der Dimension der Polysemie, die Lesarten ein und desselben Wortes involviert, verwechselt werden. Die Erscheinungen der paradigmatischen Dimension können mittels der hier interessierenden Wortfeldtheorie und/oder mit der Theorie der Sinnrelationen am besten erfaßt werden. Schließlich müssen wir noch die syntagmatische Dimension berücksichtigen, die meist Wörter aus verschiedenen Wortarten in der 'und'-Beziehung betrifft und mit wesenhaften Bedeutungsbeziehungen, Kollokationen und Assoziationen zu tun hat.

Das statische Bild unseres Diagramms verschleiert natürlich noch die innere Dynamik, die hier laufend im Gange ist. Synchron drückt sich diese Dynamik als Variation aus, diachron als Wandel. Nehmen wir die Dynamik bereits für einzelne lexikalische Einheiten in Anspruch, so gilt sie um so mehr für den gesamten Wortschatz. Der Wortschatz einer natürlichen Sprache weist eine beträchtliche kurzfristige und längerfristige Flexibilität auf. Zur kurzfristigen Flexibilität gehört die im Zusammenhang mit verschiedenen ÄuBerungen erforderliche Bereitstellung unterschiedlichster Arten von Strukturierungen, und zur längerfristigen Flexibilität gehören Veränderungen im Wortschatz insgesamt. Die hier deutlich werdende faszinierende Vielfalt der Sprache bietet die Chance für verschiedene Gliederungsprinzipien für die Frage der Abgrenzung eines relevanten Ausschnittes aus dem Allgemeinwortschatz. ${ }^{2}$

Egal wie nun der jeweilige Ausschnitt des Wortschatzes aussieht, dieser Ausschnitt selber ist wiederum für sich im Innern gegliedert. Unsere Überzeugung hierüber speist sich aus Erkenntnissen zum individuellen Wortschatz, wobei die relevanten Erkenntnisse aus der Fehlerlinguistik, der Psycholinguistik und der klinischen/medizinischen Linguistik stammen. Alles hieraus deutet auf eine ziemliche Komplexität der Gegliedertheit hin. Trivialerweise ist z.B. nicht jedes Wort mit einzig seinen Feldnachbarn innerhalb eines vorgegebenen inhaltlichen Rahmens verbunden. Verbindungen müssen ebenso über Feldgrenzen hinweg bestehen und, mindestens aufgrund der assoziativen Verknüpfungen, zwischen Wörtern verschiedener Wortarten bzw. syntaktischer Kategorien.

2 Man behilft sich hier gern für die Benennung der Unterteilungen mit dem griech. dia- 'durch'. 
Vergleichen Sie zur Veranschaulichung folgendes Beispiel zu stehlen als Transferverb im Diagramm 2: ${ }^{3}$

Syntagmatische Verbindungen für stehlen 'Transfer':

Sprichwörtl. wer stiehlt, der trugt; wer trigt, der lugt

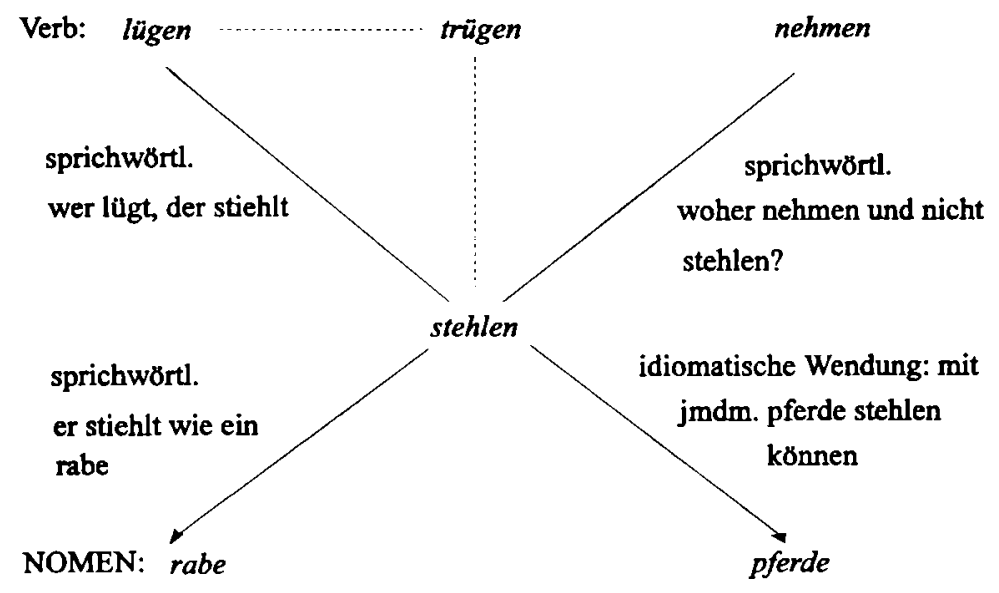

... Verbindung im aktiven Wortschatz

... falls trügen 'täuschen', 'irreführen' nur im passiven Wortschatz mit belebten Denotaten als nominalem Argument, nur Verbindung im passiven Wortschatz

$3 \mathrm{Vgl}$. für stehlen - trügen - lügen und stehlen - rabe: Wander (1876), Sp. 799, 802, 803; für stehlen - pferde: Röhrich (1973, S. 1005); für stehlennehmen: Paul (1992, S. 845). 
Paradigmatische Verbindungen für stehlen 'Transfer':

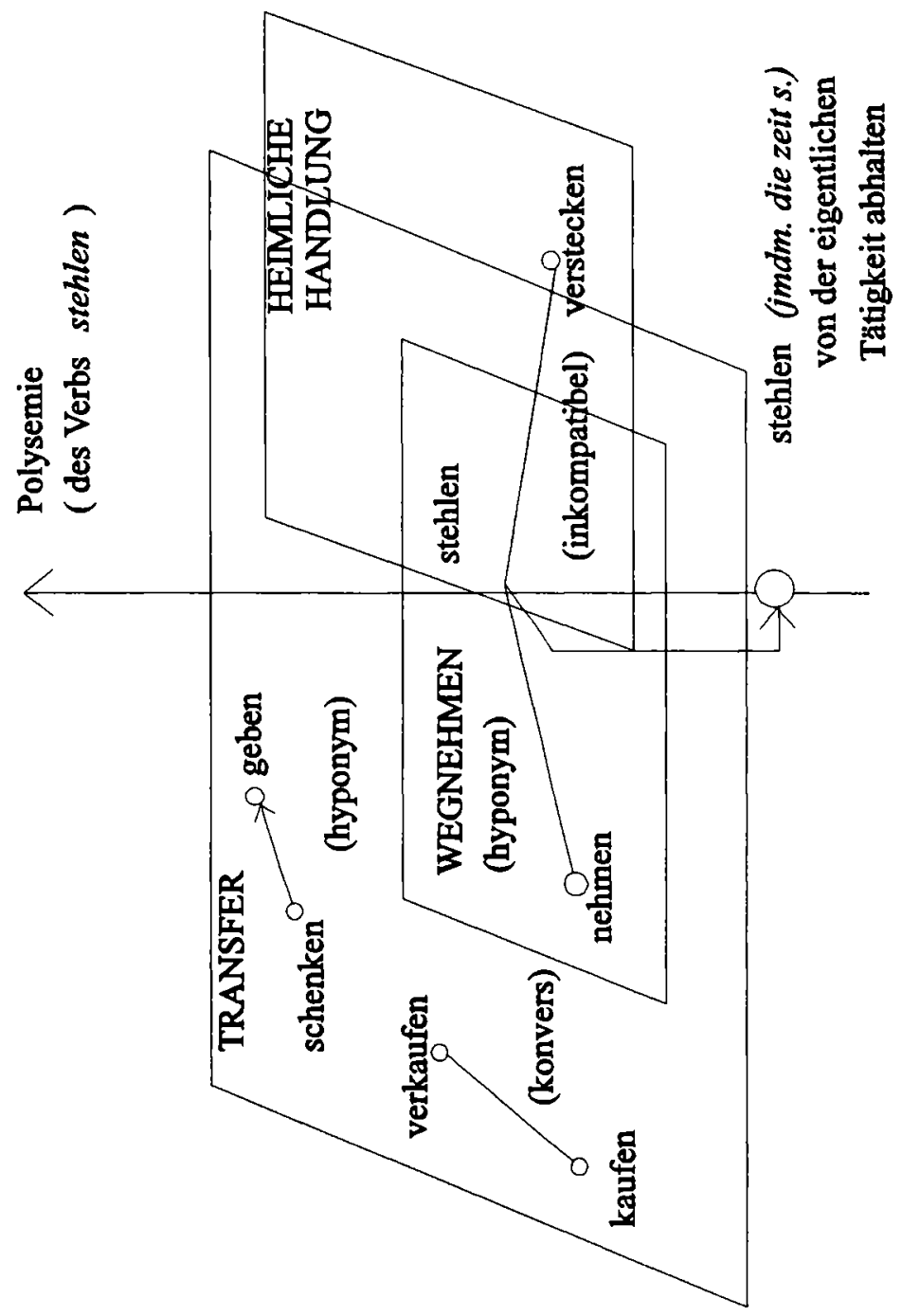


Das Paar <stehlen, nehmen> zeigt, daB paradigmatische und syntagmatische Beziehungen nebeneinander bestehen können. Zusätzlich zu dem gebotenen Bild müssen wir annehmen, daß die Gegliedertheit des Wortschatzes sich über die Zeit hinweg verändern kann, und zwar gilt dies z.B. für den individuellen Wortschatz sicherlich nicht nur für die Phase des Spracherwerbs.

Nun ist meine Betonung der unterschiedlichsten Arten von Gegliedertheiten nicht ohne Relevanz für die Einschätzung der Wortfeldtheorie. Wortfelder können nichts anderes sein als spezielle Gruppierungen im Wortschatz neben anderen Möglichkeiten. Ganz im Geiste der Wortfeldkonzeption selbst müssen Wortfelder sozusagen ihren eigenen Platz im System der Gegliedertheiten des Wortschatzes finden. Dieser eigene Platz ergibt sich in Abgrenzung und Differenzierung zu anderen Konzeptionen. D.h. aber, daB denkbare und immer wieder vorgeschlagene Erweiterungen der klassischen Vorstellungen nicht von vornherein wünschenswerte Entwicklungen darstellen, sondern jeweils erst im einzelnen mit Bedacht zu bewerten sind. Eine Entscheidung gegen eine Erweiterung darf dann nicht einfach mit einem Schwachpunkt der Konzeption gleichgesetzt werden.

Im übrigen möchte ich von der psychologischen Realität der hervorgehobenen Gegliedertheit des individuellen Wortschatzes ausgehen. Dabei meine ich mit "psychologischer Realität” folgendes: Die der Sprachfähigkeit zugrundeliegende Organisation im Gehirn läBt Gegliedertheiten der besprochenen Art auf den für die Sprachproduktion und/oder Sprachrezeption relevanten Repräsentationsebenen zu, wobei diese Gegliedertheiten wohl eher von zeitlich beschränkter Natur sind. Diese zeitliche Beschränktheit ist sozusagen Voraussetzung für die angesprochene kurzfristige Flexibilität im Wortschatz. Von „physiologischer Realität” würde ich dagegen sprechen, falls wir folgendes behaupten könnten: Die der Sprachfähigkeit zugrundeliegende Organisation im Gehirn, die relativ stabil angenommen wird, beinhaltet selbst Gegliedertheiten der besprochenen Art. Nun wissen wir wahrlich wenig über Gegebenheiten auf der Ebene physiologischer Realität. Immerhin, die Idee der Identität kann z.B. über die Ebene psychologischer Realität hinaus auf die Ebene der physiologischen Realität verlagert werden. Wir schlieBen dies aus der Fähigkeit unseres Körpers, zwischen "sich selbst” und „nicht sich selbst” unterscheiden zu können, was auf der einen Seite die problematischen Abwehrreaktionen bei Transplantationen auslöst und auf der anderen Seite das Heranwachsen des Fötus in der Gebärmutter als bisher medizinisch unerklärtes Phänomen erscheinen läßt. 


\section{Was waren Wortfelder?}

Obwohl sich sicherlich in der Geschichte der Sprachwissenschaft immer wieder vereinzelt feldhafte Vorstellungen in dem zu behandelnden Sinne auffinden lassen würden, scheint das Ende des letzten Jahrhunderts und der Beginn unseres Jahrhunderts besonders günstig für solche Vorstellungen gewesen zu sein. Dieser Eindruck entsteht, weil plötzlich in einer geballten Fülle von verschiedenen Autoren Feldgedanken vertreten werden. Wenn wir eine zweifellos vergröbernde Verallgemeinerung zulassen, dann muB als dominierendes Vorgehen in der lexikalischen Semantik zuvor die isolierte Einzelwortforschung gesehen werden.

Als Klammer für feldhafte Vorstellungen möchte ich die Überzeugung werten, daB normalerweise eine isoliert betriebene Einzelwortforschung nicht allzuviel zur Inhaltsfrage des betreffenden Wortes beitragen kann und daB somit die meisten Wörter in einem größeren Zusammenhang innerhalb des Wortschatzes zu untersuchen sind. Feldhafte Vorstellungen wenden sich allgemein gesprochen gegen ein atomistisches Vorgehen, sie setzen sich für ein ganzheitliches Vorgehen im Wortschatz ein.

Am Ende des letzten Jahrhunderts sollte neben dem von Coseriu völlig zu Recht als Vorläufer feldhafter Vorstellungen benannten Karl Wilhelm Ludwig Heyse ${ }^{4}$ zumindest auch Carl Abel Erwähnung finden. In verschiedenen Arbeiten der 80er Jahre finden wir bei ihm ganz erstaunliche relationale, feldhafte Ansichten, die immer wieder die Notwendigkeit eines Ausgreifens auf größere Ausschnitte im Wortschatz betonen. Um Ihnen ein Gefühl hierfür zu geben, sei folgendes Zitat angeführt, das aus einer Sammlung seiner Oxforder Vorlesungen stammt:

„To fully realise the pervading thoughts of a people in language, we shall (...) have to group them according to their subject-matter. If desirous to find out what the English think about freedom, we shall have to investigate the meaning of 'freedom', 'liberty', 'independent', 'selfdependent', 'uncurbed', 'unrestricted', 'unrestrained', 'unbound', 'untrammeled', \& c. We shall have to compare these several meanings and ascertain in what they supplement or restrict each other. Finally, it will be necessary in a general summary to indicate the proper position of each shade of meaning in the concept, and thus present the complete idea in a clear and classified digest." (S. 162).

Alles was unter den zu untersuchenden Begriff, den angenommenen Rahmen paßt, muß hiernach untersucht und voneinander differenziert werden. Insofern finden wir bei Abel auch nicht die Beschränkung auf

4 Vgl. Coseriu (1979). Coseriu bezieht sich auf Heyse (1856).

5 Vgl. Abel (1882). 
eine einzelne Wortart. Allerdings hatte er seine Vorstellungen vorher in Ansätzen bereits exemplifiziert, und zwar ging es in Abel (1878) um die englischen Verben des Befehls. Hierbei konzentrierte er sich dann doch auf eine einzige Wortart.

Folgende Sprachwissenschaftler, die mehr oder weniger explizit in unserem Jahrhundert feldhafte Vorstellungen vertreten haben, sind nun vor oder gleichzeitig zu Jost Trier zu nennen:

1910: Richard M. Meyer. Bei ihm ist von „Bedeutungssystemen” die Rede, wobei er unter einem Bedeutungssystem folgendes versteht (Meyer 1910a): „(...) die Zusammenordnung einer begrenzten Anzahl von Ausdrücken unter einem individuellen Gesichtspunkt." (S.359). Zwei Punkte möchte ich hiervon herausgreifen: 1. Die Betonung der Begrenztheit soll wohl über die Endlichkeit hinaus auf die Überschaubarkeit der entsprechenden Gruppierungen hinweisen. Dies ist ein sehr wohl beachtenswerter Ratschlag, gerade in der Anfangsphase einer Wortfelduntersuchung. Da die Paradigmen meist rasch anschwellen - falls man sich auf die verbalen Kontexte wirklich einläßt -, orientiert man sich anfangs besser an ausgewählten Teilmengen. 2. Aus dem "individuellen Gesichtspunkt" als Sammlungsprinzip läßt sich wahrscheinlich ableiten, daß Felder unter den verschiedensten Gesichtspunkten denkbar sind. Damit wird wohl die von uns betonte vielfältige Gliederung des Wortschatzes angesprochen und steht im Gegensatz zu der von Trier vertretenen Meinung, da $B$ der Wortschatz sich relativ undynamisch in Feldern ausgliedere. Die Aufgabe der lexikalischen Semantik charakterisiert dann Meyer (1910a) folgendermaßen: „Die Semasiologie hat für jedes Wort erstens festzustellen, welchem Bedeutungssystem (oder: welchen Bedeutungssystemen) es angehört; zweitens, welches der systembildende, differenzierende Faktor dieses Systems ist."(S. 359). Hier sollte folgendes festgehalten werden: Meyer läßt zu, daß ein Wort in mehreren Feldern vorkommen kann. Dies ist keineswegs eine Selbstverständlichkeit bei anderen Konzeptionen. Der zweite Teil ist dagegen sicherlich zu vereinfacht gesehen. In solchen Systemen können mehrere differenzierende Faktoren am Werke sein. Andererseits gehört zweifellos zu jeder Wortfelduntersuchung die Ermittlung der semantischen Struktur des Feldes. Schließlich finden sich bei Meyer (1910b) bereits Anklänge an die Mosaikvorstellung, zumindest in Form eines Puzzles: „(...) daB jede in einem einzelnen Dialekt vorhandene Bezeichnung die übrigen mitbestimmt, wie in einem Zusammenspiel jedes Steinchen die Lage aller anderen mitentscheidet." (S. 155f.).

1924: Gunther Ipsen. Bei ihm taucht in einer onomasiologischen Arbeit zu den indogermanischen Bezeichnungen von Metallen wohl zum ersten 
Mal der sprachwissenschaftlich zu verstehende Begriff 'Feld' auf. Vgl. Ipsen (1924, S. 225):

„(...) die Eigenwörter [im Unterschied zu den Lehnwörtern. P.R. Lutzeier] stehn in einer Sprache nie allein, sondern sind eingeordnet in Bedeutungsgruppen (...). Diese Verknüpfung ist aber nicht als Aneinanderreihung an einem Assoziationsfaden gemeint, 'sondern so, daB die ganze Gruppe ein 'Bedeutungsfeld' absteckt, das in sich gegliedert ist, wie in einem Mosaik fügt sich hier Wort an Wort, jedes anders umrissen, doch so, daß die Konturen aneinanderpassen und alle zusammen in einer höheren Ordnung auf-(...)gehen."

Mit 'Feld' ist also hier in erster Linie die Gliederung im Inhaltsbereich gemeint, wobei die danach auch von Trier aufgegriffene und berechtigterweise immer wieder scharf kritisierte Mosaikmetapher ebenfalls zur Sprache kommt und mit 'Wort' eigentlich genauer 'Wortinhalt' gemeint sein muB.

1934: André Jolles. Jolles beruft sich bei seinen Überlegungen auf Vorstellungen von Dionysios Thrax, also auf Vorstellungen eines griechischen Grammatikers aus dem 2. Jh.v.Ch., und spricht selbst auch von „Bedeutungsfeldern”. Jene sind jedoch denkbar minimale Felder, da er einzig Oppositionspaare als Repräsentanten ansetzt. Z.B.: vater - sohn, tag - nacht und links - rechts. Im Unterschied zur einseitigen Gerichtetheit bei den von Porzig (1934) eingeführten 'wesenhaften Bedeutungsbeziehungen' geht Jolles von einer gegenseitigen Bestimmung der beiden Elemente aus. Es ist sicherlich kein Fehler, von Oppositionspaaren bei einer Wortfeldbeschreibung auszugehen. ${ }^{6}$ Nur, falls es uns bei der Wortfeldkonzeption um die Erfassung von relevanten Ausschnitten des Wortschatzes geht, sollte auch klar sein, daß man hierbei nicht stehenbleiben darf.

Schließlich komme ich zu Jost Trier: Das wohl bekannteste Werk vom Titel oder zumindest vom Hörensagen her der Wortfeldforschung überhaupt ist sicherlich: „Der deutsche Wortschatz im Sinnbezirk des Verstandes. Die Geschichte eines sprachlichen Feldes. Band 1 (Von den Anfängen bis zum Beginn des 13. Jahrhunderts). Heidelberg: Carl Winter 1931 (2. Auflage 1973)". Trier beruft sich hier unter anderem auf Saussure. Dabei überwindet allerdings Trier die von Saussure sicherlich zu starr angesetzte Trennung zwischen Diachronie und Synchronie, denn, es wird mit dem Feldgedanken zwar der vordringlich synchrone Systemgedanke aufgegriffen, gleichzeitig aber dieses System auch in seinen Veränderungen gezeigt. Ferner setzt sich Trier explizit von dem bis

6 Ähnliches empfiehlt auch Coseriu in seinen Arbeiten. 
dahin üblichen Untersuchungsinteresse der Onomasiologie ab, d.h. eine Bezeichnungsgeschichte der Sichel, des Pfluges usw. zu liefern. Nicht die Bezeichnungsgeschichte individueller Dinge bzw. der entsprechenden Begriffe ist von nun an von Interesse, vielmehr geht es um die Bezeichnungsgeschichte von ganzen Inhaltskomplexen, die, um mit Meyer zu sprechen, über „individuelle Gesichtspunkte” festgelegt sind. Trier beginnt nun seine berühmte Abhandlung folgendermaßen (Trier 1931, S. 1):

„Kein ausgesprochenes Wort steht im BewuBtsein des Sprechers und Hörers so vereinzelt da, wie man aus seiner lautlichen Vereinsamkeit schließen könnte. Jedes ausgesprochene Wort läft seinen Gegensinn anklingen. Und noch mehr als dies. In der Gesamtheit der beim Aussprechen eines Wortes sich empordrängenden begrifflichen Beziehungen ist die des Gegensinns nur eine und gar nicht die wichtigste. Neben und über ihr taucht eine Fülle anderer Worte auf, die dem ausgesprochenen begrifflich enger oder ferner benachbart sind. Es sind seine Begriffsverwandten. Sie bilden unter sich und mit dem ausgesprochenen Wort ein gegliedertes Ganzes, ein Gefüge, das man Wortfeld oder sprachliches Zeichenfeld nennen kann. Das Wortfeld ist zeichenhaft zugeordnet einem mehr oder weniger geschlossenen Begriffskomplex (...). Dies in einem inhaltlich zusammengehörigen Teilausschnitt des Wortschatzes - eben dem Wortfeld - sichtbar werdende Gefüge ist also die äuBere, zeichenhafte Seite der begrifflichen Aufteilung (...). Die das Wortfeld, den Wortmantel, die Wortdecke mosaikartig zusammensetzenden Einzelworte legen - im Sinne ihrer Zahl und Lagerung - Grenzen in den Begriffsblock hinein und teilen ihn auf."

Hier darf man die anfängliche Rede vom Bewußtsein des Sprechers selbstverständlich nicht allzu wörtlich nehmen. Immerhin wird auch bei Trier mit dem Verweis auf den Gegensinn der Oppositionsgedanke angesprochen. Viel wichtiger ist mir jedoch, daß mit dem Begriff 'Wortfeld' nun offenbar zum ersten Male eine Gruppierung von Formen in den Blickpunkt rückt. Eine solche Gruppierung wird gleichzeitig einem gegliederten Inhaltsbereich zugeordnet. Dieser wichtige Gedanke, daß ein Feld sowohl eine Formseite als auch eine Inhaltsseite aufweist - auf einer höheren Ebene also Zeichencharakter hat, wird bei meiner eigenen Wortfeldkonzeption wiederum voll aufgegriffen. Im weiteren finden wir bei Trier (1931) die bereits als Charakteristikum feldhafter Vorstellungen bezeichneten Eigenschaften: „Die Worte im Feld stehen in gegenseitiger Abhängigkeit voneinander. Vom Gefüge des Ganzen her empfängt das Einzelwort seine inhaltliche begriffliche Bestimmtheit." (S. 2). Alle Elemente des Wortschatzes müssen offenbar Platz in irgendwelchen Feldern finden, denn „Außerhalb eines Feldganzen kann es ein Bedeuten überhaupt nicht geben." (S. 5). Betonen möchte ich noch, daß Trier (1931) 
weitere inhaltsbestimmende Faktoren durchaus anerkennt, so etwa im Satzzusammenhang (S. 4).

Das Feld ist nun für Trier Ausgangspunkt praktischer einzelsprachlicher Forschung. Seine Vorgehensweise ist dabei sammelnd, 'von unten' her am Gebrauch in Texten orientiert. Auffallend ist dabei folgendes: Die inhaltlichen Gliederungsfaktoren müssen nicht rein aus den Texten bzw. der Sprache gewonnen werden, sondern er schreibt in Trier (1931, S. 24):

(...) zu Zwecken der Feldbeschreibung - wenigstens in gröbsten Umrissen Einteilungslinien gewonnen werden müssen, die einer theoretisch strenger denkenden, einer systematischeren Welt entstammen, als sie die Mehrzahl der ahd. und mhd. Werke darstellt. Diese Einteilungslinien (...) sind aus der mittelalterlichen Philosophie zu gewinnen."

Diese keck an einer fachwissenschaftlichen Sichtweise vorgenommene Orientierung paft so gar nicht zum üblichen Vorurteil über verstaubtes, inhaltsbezogenes Arbeiten in der Feldforschung. Sicherlich muß dieses Vorgehen allerdings in diesem Falle auch hinterfragt werden. Es bliebe zu klären, inwieweit diese Kategorien mittelalterlicher Philosophie tatsächlich Relevanz für die untersuchten Textsorten hatten, ganz zu schweigen von der damaligen Sprachgemeinschaft.

Entscheidend für die praktische Arbeit und damit auch für die Attraktivität der Wortfeldkonzeption ist nun aber folgende berühmte Erkenntnis: Das Feld steht zwischen dem Einzelwort und dem gesamten Wortschatz (Trier 1934): „Felder sind die zwischen den Einzelworten und dem Wortschatzganzen lebendigen sprachlichen Wirklichkeiten, die als Teilganze mit dem Wort das Merkmal gemeinsam haben, daß sie sich ergliedern, mit dem Wortschatz hingegen, daß sie sich ausgliedern." (S. 132). Vergleichen Sie die Illustration Diagramm 3:

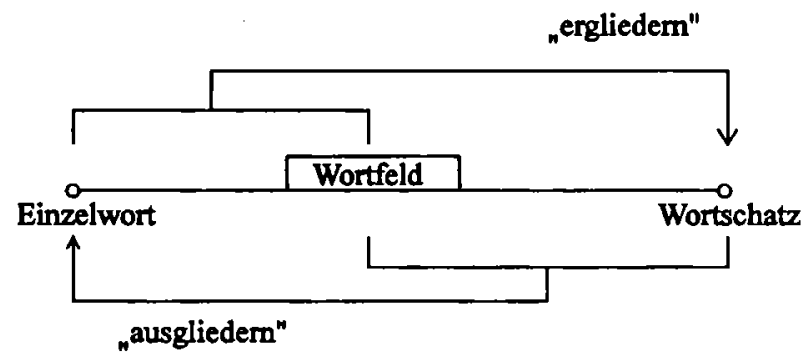

Bei einer insgesamt wünschenswerten Abkehr von der isolierten Einzelwortuntersuchung sind wir nicht auf den unüberschaubaren Wort- 
schatz als Ganzes zurückgeworfen. Es sei aber auch folgendes noch einmal erwähnt: Zwischen den beiden Polen 'Einzelwort' und 'Wortschatz' gibt es noch zahlreiche weitere strukturierte Ausschnitte. Wie gesagt, bei Berücksichtigung dieser Tatsache verfält man nicht in die Versuchung, alles der Wortfeldkonzeption aufbürden zu wollen.

\section{Was sind Wortfelder?}

Ich werde nun aus meiner Warte die gegenwärtige Konzeption so kurz wie möglich schildern. Es geht hierbei in erster Linie um die geeignete Ausformulierung folgender Feldprinzipien:

1. Prinzip: Felder involvieren global gesehen eine Rahmenbildung.

2. Prinzip: Felder als zeichenhafte Gebilde weisen eine Formseite und eine Inhaltsseite auf.

3. Prinzip: Jedes Feldelement erhält seine Bestimmung in Auseinandersetzung mit anderen Elementen des Feldes.

Das erste Prinzip in Zusammenhang mit dem zweiten Prinzip läßt eine Rahmenbildung sowohl auf der Formebene als auch auf der Inhaltsebene erwarten.

Was die Formebene betrifft, so verstehen wir in völliger Übereinstimmung mit der Tübinger Schule ${ }^{7}$ das Wortfeld als spezielle paradigmatische Gruppierung im Wortschatz. Die im ersten Prinzip angesprochene Rahmenbildung übernimmt ein verbaler Kontext, und wir erfassen damit im Paradigma die untereinander alternativen Möglichkeiten an der jeweiligen Substitutionsstelle. Abgesehen von einigen eher technischen morpho-syntaktischen Schwierigkeiten ergeben sich hier sofort zwei Fragen, deren Beantwortung die Ausgestaltung der Formebene unserer Felder entscheidend beeinflußt. Die erste Frage betrifft die morphosyntaktische Komplexität der Elemente, die wir noch als Elemente unserer Felder akzeptieren wollen. Die zweite Frage betrifft dagegen die morpho-syntaktische Variabilität, die wir in unseren Feldern dulden wollen. Sprechen wir von Wortfeldern, dann scheint mir im Hinblick auf die erste Frage klar, daß wir als Mitglieder nur Wörter erfassen wollen. Im allgemeinen wird dabei keine strikte Grenzziehung zwischen Simplizia und Wortbildungen angestrebt. Nun wissen wir aber auch, daB zu dem Bereich der Einheiten des Wortschatzes noch einige wortartige Elemente hinzugezählt werden müssen. Wir erhalten hierdurch die sog. lexikali-

7 Vgl. z.B. Coseriu (1970) oder Geckeler (1971). 
schen Elemente, die aus den Wörtern und den Phraseologismen ${ }^{8}$ bestehen. Bei dem Verständnis von Feldern als spezielle Gruppierungen im Wortschatz spricht selbst verständlich nichts gegen die Hereinnahme aller lexikalischen Elemente als Elemente unserer Felder. Zumal sich die Phraseologismen ohne größere Schwierigkeiten unter meine Konzeption subsumieren lassen, wie Schindler (1993) auf überzeugende Weise nachgewiesen hat. Allenfalls würde ich für eine terminologische Differenzierung der beiden Fälle plädieren. Sind alle lexikalischen Elemente als Feldelemente zugelassen, sollten wir von lexikalischen Feldern reden, während wir im Falle der Beschränkung auf Wörter weiterhin von Wortfeldern reden sollten. Insofern sind Wortfelder Sonderfälle der im Titel bereits angesprochenen lexikalischen Felder. ${ }^{9}$ Die bei der Ermittlung des Paradigmas auftretende morpho-syntaktische Variabilität der substituierbaren Elemente - ich komme damit zur zweiten Frage - hängt natürlich in erster Linie von dem gewählten verbalen Kontext ab. Syntagmen, die keine Phraseologismen darstellen, lassen sich über das Prinzip der Komposition ermitteln, sind also für den Feldgedanken von vornherein nicht interessant. Wie sieht es jedoch für Elemente aus, die aus verschiedenen Wortarten bzw. syntaktischen Kategorien stammen? Ich selber habe mich schon immer auch in dieser Frage eher an dem ersten Feldprinzip orientiert: Die Formseite von Feldern berücksichtigt als entscheidenden Rahmen die Grenzen der Wortarten bzw. syntaktischen Kategorien. Für Beziehungen über diese Grenzen hinweg ist meiner Meinung nach auf der Formebene in erster Linie die Wortbildung zuständig. Man denke hier nur an die Beziehungen zwischen dem Adjektiv bange aus dem Wortfeld der Adjektive für Angstgefühle und dem Substantiv bange und dem Verb bangen. Allenfalls von einer onomasiologischen Warte aus läBt sich somit an diesem Punkt für die Hereinnahme von Elementen aus den angesprochenen drei Wortarten argumentieren. Ohne auf die Behandlung der Inhaltsseite vorgreifen zu wollen, sei an dieser Stelle folgendes angeführt: So unbestritten dieser onomasiologische Gesichtspunkt seine allgemeine Gültigkeit haben mag, so wenig überzeugend muß er für den eigentlichen Feldgedanken wirken. Die Erfassung irgendwelcher systematischer Beziehungen wird ja bereits über die Interpretation der Wortbildungsprozesse erfolgen können. Die im Zusammenhang mit den Feldern speziell interessierende Position des jeweiligen Feldelementes muB dagegen in Auseinandersetzung mit den dazu in engster paradigmatischer

8 Zu den Phraseologismen zähle ich metaphorische Wendungen, idiomatische Wendungen und Wendungen mit obsoleten Wörtern.

9 Ich folge mit diesen terminologischen Vorschlägen im großen und ganzen Lipka (1980). 
Beziehung bestehenden Elementen geschehen, und dies können nur Elemente aus derselben Wortart sein. Die damit wiederum explizit akzeptierte Beschränkung des Feldgedankens läBt sich ohne größere Probleme verschmerzen, denn, wie bei Behandlung der Inhaltsebene gleich noch einmal betont werden wird, die Position des Elementes auf der Inhaltsebene des Feldes schöpft im allgemeinen die jeweilige Lesart des Elementes sowieso nicht voll aus.

Kommen wir damit zur Inhaltsebene unserer Felder. Im Hinblick auf die Elemente unserer lexikalischen Felder muß die semantische Ähnlichkeit untereinander garantiert sein. Dies ist nichts anderes als die Garantie der im ersten Feldprinzip ausgedrückten Rahmenbildung. Zur Illustration der Überlegungen an dieser Stelle verweise ich auf den verbalen Kontext: $\alpha=$ das buch erweist sich als $x$. Legen wir uns gleich auf die syntaktische Kategorie $\mathbf{c}=$ 'Adjektiv' fest, dann erhalten wir etwa folgendes Paradigma $\mathbf{P}=\{$ alt, interessant, umfangreich, originell, dick, langweilig, teuer, langatmig, neu\}. Dieses Paradigma ist offensichtlich inhaltlich immer noch ziemlich heterogen. Im nächsten Schritt muB es daher um die Angabe einer inhaltlichen Klammer für die zu untersuchenden Elemente gehen. Ich spreche hier von der Angabe eines Aspektes, der den Trierschen Sinnbezirk abstecken soll. Zwei Möglichkeiten für Aspekte bieten sich für unser Beispiel unmittelbar an: 1. A = 'Art des Erwerbs': Dies führt auf das Paradigma $\mathrm{P}^{\prime}=\{$ alt, teuer, neu $\} .2 . \mathrm{A}=$ 'Bewertung des Inhalts': Dies führt auf das Paradigma P" = \{interessant, originell, langweilig, langatmig, neu\}. Solche Aspekte geben allgemeine Bedeutungen vor, und die Lesarten der Elemente im fraglichen Paradigma müssen zu diesem Aspekt passen; genauer, es muß solche Lesarten der Elemente geben, die als Spezifizierungen des Aspekts verstanden werden können. Selbstverständlich können wir nicht erwarten, daB im Paradigma jeweils selber ein Element zu finden ist, für das der gerade noch zu akzeptierende Grenzfall eintritt, nämlich der Fall, bei dem eine Lesart des Elements mit dem Aspekt zusammenfältt. ${ }^{10}$ Die Forderung eines Aspektes ist unser erstes echtes semantisches Kriterium: Der Aspekt setzt den relevanten Rahmen auf der Inhaltsebene. Ähnliche Forderungen sind in der Wortfeldtheorie wohlbekannt. So lesen wir bei Coseriu (1973, S. 49) von einem Feldwert und bei Meyer war von dem "individuellen Gesichtspunkt" die Rede gewesen. So wichtig dieses Kriterium zweifellos ist, wir dürfen auf die Berücksichtigung der Kriterien für die Formebene nicht verzichten, um nicht einfach auf der Stufe rein onomasiologischer Paradigmen zu

10 Bei Coseriu (1967) heißen solche Elemente Archilexeme, anscheinend unabhängig davon, ob es dafür tatsächlich eine Form gibt oder nicht. Insofern wäre in seinem Ansatz wohl die Redeweise von 'Archisemem' konsequenter. 
verharren. Ferner verlieren wir durch die Berücksichtigung der Formebene nicht die Sprachwirklichkeit aus dem Auge, bei der uns lexikalische Elemente ja nur im Äußerungskontext erfahrbar sind. Da nun ein inhaltlicher Aspekt vorgegeben ist, treten die lexikalischen Elemente als Einheit von Form und Inhalt in unsere Felder ein. ${ }^{11}$

Die inzwischen ermittelten paradigmatischen Gruppierungen stellen sicherlich Kandidaten für Felder dar. Ob sie es aber auch wirklich sind, hängt von der Erfüllung des dritten Feldprinzips ab. Bezugspunkt hierfür kann nur die zu fordernde inhaltliche Struktur eines Feldes sein - das, was letztlich mit der Gegliedertheit des Feldes gemeint ist. ${ }^{12}$ Die Annahme einer inhaltlichen Struktur für das Wortfeld ist ebenfalls ein traditionelles Unterfangen. Trier sprach vom "Gefüge des Feldes", Ipsen dachte an ein Mosaik, und Weisgerber (1951/52) benützte die Redeweise von der „innersprachlichen Struktur”. Als Instrumentarium zur Erfassung der fraglichen Struktur benütze ich sowohl Dimensionen als auch Sinnrelationen.

Was die Dimensionen angeht, so gelten hier die folgenden Ziele: Einem vorgegebenen Paradigma sollen endlich viele Dimensionen zugeordnet werden. Jede einzelne Dimension bewirkt dabei eine Zerlegung des Paradigmas. Die bei einer durch die Vorgabe einer Dimension bewirkte Zerlegung des Paradigmas führt somit auf untereinander disjunkte Teilmengen. Diese Teilmengen erhalten nun einen Namen. Diese Namen können nicht völlig beliebig gewählt werden, da ihnen eine gewisse Rolle für die Inhaltsebene von unseren Feldern zugedacht ist. Stellen wir uns etwa das Wörter-Paradigma der Substantive unter dem Aspekt A = 'Gewässer' vor. Dieses Paradigma umfaBt sicherlich die Substantive: bach, fluß, kanal, meer, rinnsal, see, gewässer, strom, teich, tümpel. Eine anzusetzende Dimension D 1 ('Frage der Bewegung') bewirkt die Teilmengen T1, T2, T3, wobei zu T1 etwa bach, fiuß, kanal, rinnsal, strom, zu T2 etwa meer, see, teich, tümpel und zu T3 gewässer zählen würden. Wenn wir nun zunächst den Namen 'fließend' für T1 und den Namen 'stehend' für T2 vorsehen, können Sie wohl leicht erraten, worum es bei dieser Namensge-

11 Schlaefer (1987, S. 175), der die Vorgabe eines Aspektes angeblich überflüssig machen will, indem er bei seiner Untersuchung von einzelnen Wörtern und ihren Umgebungen ausgeht, setzt selbstverständlich mit grinsen usw. den durch diese Formen vermittelten Inhaltsbereich. Ähnlichkeitsund Wohlgeformtheitsbeziehungen, ob er es will oder nicht, werden in diesem Fall durch seine Intuition beurteilt. Becker (1991, S. 100), der eigentlich ansonsten mit dem Vorgehen von Schlaefer sympathisiert, hat dies klar erkannt.

12 Wir können hier auch vom semantischen Feld sprechen. 
bung für die Zerlegungsmengen geht: Aus einer intuitiv vorzunehmenden Paraphrasierung der Bedeutung von etwa bach unter dem Aspekt $\mathbf{A}=$ 'Gewässer' würde sich unter anderem inhaltsmäBig normalerweise so etwas wie 'fließendes Gewässer' ergeben. Mit anderen Worten, der Name 'fließend' gibt normalerweise einen notwendigen Anteil an der Bedeutung von bach unter dem Aspekt A = 'Gewässer'. Verallgemeinert können wir somit den Namen der Zerlegungsmengen folgenden Status zusprechen: Die Namen der Zerlegungsmengen einzelner Dimensionen repräsentieren normalerweise notwendige Anteile an der Bedeutung der in den jeweiligen Zerlegungsmengen vorkommenden Elemente bezüglich des vorgegebenen Aspekts. Wie im Falle von T3 mit gewässer bereits erkennbar ist, müssen wir noch Spezialfälle für die Namensgebung berücksichtigen. Und zwar gibt es zwei solche Fälle, für die wir die Namen 'Neutral', relevant z.B. für T3, und 'Weder noch' reservieren. ${ }^{13}$ Es seien zur Verdeutlichung die unterschiedlichen Funktionen einer Dimension und der Namen für die auftretenden Teilmengen besonders hervorgehoben: Die Dimension bewirkt eine Zerlegung des Paradigmas, d.h., hier ist ein formaler Blickwinkel vorherrschend, nämlich die vollständige Verteilung der Elemente auf die Teilmengen. Auf dieser Stufe ist dann auch für D1 die Teilmenge T3 bei unserem Gewässerbeispiel völlig gleichberechtigt zu den beiden anderen Teilmengen. Die Unterschiede werden erst über die Namen der Teilmengen verdeutlicht, wobei diese Namen mit den Lesarten der Elemente aus den entsprechenden Teilmengen in erklärter Weise in Verbindung gebracht werden können. Deshalb berühren die Namen auch die Inhaltsebene der Elemente für den vorgegebenen Aspekt. Daß es bei den Dimensionen um eine Zerlegung des Paradigmas gehen muß, und es sich schon gar nicht um eine Zerlegung möglicher Gegebenheiten in der außersprachlichen Wirklichkeit handeln kann, sieht man an folgendem, extremen Paradigma: $\mathrm{P}=$ \{venus, morgenstern, abendstern $\}$. Alle drei Substantive weisen ein und dieselbe Extension auf, nämlich die Einerklasse, die aus der Venus besteht. Bei diesem Stern gibt es also nichts zu zerlegen, dennoch könnte eine Dimension in Anlehnung an Freges "Sinn"-Unterschiede ${ }^{14}$ D ('Frage des Zeitpunktes des Auftretens am Himmel') eine Zerlegung unseres Paradigmas in die Teilmengen $\mathrm{T} 1=$ $\{$ morgenstern $\}$ mit dem Namen N1 = 'der am Morgenhimmel am hellsten leuchtende Stern', T2 = abendstern mit dem Namen N2 = 'der am Abendhimmel am hellsten leuchtende Stern' und T3 = venus mit dem Namen N3 = 'Neutral' bewirken.

13 Für mehr Informationen hierüber vgl. Lutzeier (1981, S. 114-115). 
Die beim üblichen Verfahren der Merkmals-/Komponentialanalyse auftretenden Merkmale sind letztlich auch Kandidaten, die zur Paraphrasierung in meinem Sinne herangezogen werden können. Allerdings ist hier ein kleiner, aber nicht unwichtiger Unterschied zu beachten, nämlich die bewuBte Einschränkung bei meinen Formulierungen auf 'normalerweise'. Was ist darunter zu verstehen? Wenn ich 'normalerweise' in meinen Formulierungen über den Status der Namen benütze, dann gehe ich von der Annahme aus, daf aufgrund der Angabe des Aspektes und der Dimension ein Stereotyp spezifiziert wird, das für die jeweilige Paraphrasierung herangezogen wird. Außerhalb der Grenzen dieses Stereotyps ist selbstverständlich nicht mehr sicher, ob die gefundenen Namen noch notwendige Anteile an der Bedeutung des Elementes repräsentieren. Wichtig ist weiter zu erkennen, daß die Dimensionen über die Namen der Zerlegungsmengen nicht die Bedeutung der Elemente des Wortfeldes bezüglich des vorgegebenen Aspektes konstituieren. Deshalb ist es auch nicht weiter schlimm, wenn eine Person zu verschiedenen Zeiten oder verschiedene Personen zur gleichen Zeit zu unterschiedlichen Dimensionen und $\mathrm{Na}$ men ihrer Zerlegungsmengen für ein und dasselbe Paradigma kommen. In der Tat ist nichts anderes zu erwarten: Bei der Paraphrasierung der Bedeutung der Elemente ergeben sich vielerlei Dinge, die auf den ersten Blick allesamt Kandidaten für Dimensionen und Namen sein können. Neben den besprochenen Anforderungen an die Dimensionen und Namen und dem persönlichen Interesse kann man noch folgende weitere Kriterien zur Entscheidung zwischen mehreren Möglichkeiten für Dimensionen heranziehen: 1. Diejenige Dimension ist vorzuziehen, die nicht nur hilft, einzelne Elemente auszusondern, sondern durchgängig eine Unterteilung in mehrelementige Mengen leistet. Man kann dann davon ausgehen, daB die betreffende Dimension grundlegendere Inhaltszüge anspricht. 2. Diejenige Dimension ist vorzuziehen, die nicht nur eine Unterteilung einer bereits bestehenden Zerlegungsmenge durchführt, sondern die über durch andere Dimensionen eingeführte Grenzen hinweg eine Unterteilung bewirkt. Bei einem solchen Fall spricht man häufig von Kreuzklassifikationen. Auch in diesem Fall kann man von der neuen Dimension neue Gesichtspunkte erwarten. 3. Diejenige Dimension ist vorzuziehen, die eine Unterscheidung mit sich bringt, die nicht oder zumindest nicht so einfach über Sinnrelationen zu erreichen wäre. Geht es um die Erfassung gradueller Abstufungen, dann scheint es generell von Vorteil zu sein, eine Dimension anzusetzen. Offensichtlich ist hier die Frage des Zusammenspiels zwischen beiden Grundpfeilern der inhaltlichen Struktur unserer Felder, nämlich zwischen den Dimensionen und den Sinnrelationen angesprochen. Aus meiner Charakterisierung der Rolle der Dimensionen für die Strukturierung eines Wortfeldes sollte man folgende Lehre ziehen: 
Anstatt von den Dimensionen als Beschreibungsinstrument allzuviel zu verlangen, was traditionellerweise immer getan wurde, schauen wir uns besser nach etwas anderem um. Dies ist keinesfalls im Sinne einer die Dimensionen ausschließenden Alternative gemeint, sondern im Sinne einer fruchtbaren Ergänzung. Diese Ergänzung muß sich insbesondere für die Strukturierung von Wortfeldern bewähren. Bekanntermaßen stellen nun die Sinnrelationen ein Instrumentarium zur Verfügung, das erlaubt, gewisse Gliederungen im Wortschatz zu erfassen. Da es sich auch hier um paradigmatische Gruppierungen handelt, liegt es nahe, dieses Mittel auch für unsere Wortfelder heranzuziehen.

Sinnrelationen bestehen in erster Annäherung zwischen lexikalischen Elementen im Wortschatz. Wie der Name 'Sinnrelation' andeutet, wird man erwarten, daB die betreffenden Relationen zwischen bestimmten Elementen aufgrund näher zu präzisierenden Beziehungen zwischen den Bedeutungen dieser Elemente bestehen. Folgendes kann nun nicht genug betont werden: Die zu erfassenden Beziehungen können nicht pauschal für 'die' Bedeutungen angesetzt werden, auch hier brauchen wir eine Relativierung auf Aspekte. Diese für die Sinnrelationen erforderliche Rahmenbildung paßt selbstverständlich zu unserem allgemeinen Vorhaben: Da unsere Felder eine entsprechende Relativierung auf Aspekte verlangen, sollte die Integration der Sinnrelationen in die Beschreibung der inhaltlichen Struktur der Felder keine Schwierigkeiten bereiten.

Als allgemeines Format für unsere Sinnrelationen ergibt sich folgendes:

Gegeben seien: A .... Aspekt, $\mathrm{l}_{1}=\left\langle\mathrm{f}_{1}, \mathrm{i}_{1}\right\rangle, \mathrm{l}_{2}=\left\langle\mathrm{f}_{2}, \mathrm{i}_{2}\right\rangle \ldots$ lexikalische Elemente, $\left.\mathrm{i}\right|_{\mathbf{A}} \ldots$ Inhalt $\mathrm{i}$ relativiert auf Aspekt $\mathrm{A}$.

$$
<<\mathrm{f}_{1}, \mathrm{i}_{1}>,\left\langle\mathrm{f}_{2} \mathrm{i}_{2}>, A>\left.\left.\in \mathrm{SR} \mathrm{gdw} \mathrm{i}_{1}\right|_{\mathrm{A}} \mathrm{R} \mathrm{i}_{2}\right|_{\mathrm{A}}\right. \text {. }
$$

Als Aufgabe bei den einzelnen Sinnrelationen stellt sich dann, die charakterisierende Beziehung $\mathrm{R}$ zwischen den relevanten Lesarten zu spezifizieren. Prinzipiell können wir eine Unterteilung bei den Sinnrelationen vornehmen, je nachdem, ob sie an einer 'vertikalen' Strukturierung oder an einer 'horizontalen' Strukturierung beteiligt sind. Die 'vertikalen' Strukturprinzipien führen auf hierarchieähnliche Gebilde im Wortschatz. Sinnrelationen, die hierzu gehören sind: die Hyponymie-Relation und die Partonymie-Relation. Für die 'horizontalen' Strukturierungen ist, abgesehen von der Synonymie-Relation, eine Ausprägung des Bedeutungsgegensatzes vorherrschend. Zu den Sinnrelationen in diesem Bereich zählen die Inkompatibilitäts-Relation, die Antonymie-Relation, die Komplementaritäts-Relation, die Konversen-Relation und die Reversivitäts-Relation. Da die Partonymie-Relation innersprachlich gesehen von nur untergeordnetem Interesse ist und die einen Bedeutungsgegensatz 
betreffenden Relationen meist Sonderfälle der Inkompatibilitäts-Relation darstellen, kann man sich übrigens bei einer Feldanalyse zunächst auf die Hyponymie-Relation und die Inkompatibilitäts-Relation konzentrieren. Die angesprochenen Sinnrelationen ${ }^{15}$ sollten als Ergänzung zu den Dimensionen verstanden werden, und zwar im Hinblick auf die Frage der zu fordernden Strukturiertheit der Inhaltsebene unserer Felder. Das Ziel hierfür ist die Erfüllung des dritten Feldprinzips: Jedes Element im Feld muß seine eigene Position erhalten. Wobei für uns diese eigenständige Position nicht allein durch die Dimensionen zu garantieren ist, sondern auch über das Netz an semantischen Beziehungen zu den anderen Elementen im Feld erfolgen kann. Die Position eines Elementes muB damit als vernünftiges Zusammenspiel zwischen den Teilmengen, denen das Element aufgrund der angesetzten Dimensionen angehört, und den Sinnrelationen zu den anderen Elementen verstanden werden. Die Präzisierung dieses Gedankens kann selbstverständlich nur im Rahmen einer Definition für den Begriff 'Wortfeld' bzw. 'lexikalisches Feld' erfolgen. Da diese hier nicht gegeben werden kann, erlauben Sie mir, wenigstens einige Bemerkungen hierzu anzuführen. ${ }^{16}$

1. Wenn wir die Aufgaben der Dimensionen mit den dazugehörigen $\mathrm{Na}$ men mit den Aufgaben der Sinnrelationen vergleichen, dann stellen wir folgenden wichtigen Unterschied im Hinblick auf die Ermittlung der relevanten Lesart eines Elementes fest: Ein direkter, unmittelbarer Bezug auf die Anteile der Lesart ist allenfalls über die Namen der Teilmengen möglich, Sinnrelationen als Ausdruck von eher innersprachlichen Beziehungen $z$ wischen Lesarten können dies nicht leisten. Die in Form der Sinnrelationen ausgedrückten Beziehungen verlangen nach einem Anker nach außen. ${ }^{17}$

2. Die Hereinnahme der Sinnrelationen erlaubt eine elegante Lösung der ansonsten leidigen Vollständigkeitsfrage der Paradigmen. Intuitiv gesehen gehört ein Element zum Feld, falls es in den geforderten Rahmen auf der Formebene und in den geforderten Rahmen auf der Inhaltsebene paßt. Die Operationalisierung dieser Überlegung läuft dann, was die problematischere Inhaltsebene angeht, über die Überprüfung der Abgeschlossenheit gegenüber den semantischen Beziehungen für den vorgegebenen Aspekt. Insgesamt führen wir hier 3 hinreichende Bedin-

15 Fūr mehr Informationen hierzu vgl. Lutzeier (1981, S. 122ff.) und (1985b).

16 Eine Definition findet sich z.B. in Lutzeier (1981, S. 138ff.).

17 Eine ausführliche Diskussion dieser Gedanken findet sich in Lutzeier (1983b). 
gungen für die Mitgliedschaft im Felde an: Entweder tritt das fragliche Element in die Inkompatibilitäts-Relation mit einem Element des Wortfeldes bezüglich des vorgegebenen Aspektes ein, oder es ist einem Element des Wortfeldes bezüglich des vorgegebenen Aspektes im Sinne der Hyponymie-Relation unter- oder übergeordnet. Irgendwelche isoliert auftretende Elemente, die unter den allgemeinen Aspektrahmen fallen, können separat behandelt werden, da wir die Bedingungen nicht als notwendig verstehen. Diese Offenheit gegenüber solchen isolierten Elementen scheint mir für unsere Konzeption durchaus gerechtfertigt, insbesondere, wenn man sich an unsere Charakterisierung feldhafter Vorstellungen erinnert: Da ging es in erster Linie um die Sicht der Abhängigkeit zwischen den einzelnen Elementen im Wortschatz. Für isolierte Elemente gilt nun eine solche Abhängigkeit offensichtlich gerade nicht. Insofern müssen unsere Felder nicht unbedingt die bei vorgegebenem Aspekt maximal möglichen Mengen darstellen. Dies verdeutlicht wiederum den Unterschied zu Gruppierungen, die unter einem rein onomasiologischen Gesichtspunkt gewonnen werden. Sobald wir allerdings die Existenz eines Archilexems vorfinden, erhalten wir über die entsprechende Hyponymie-Relation automatisch das maximale Paradigma. Der angestrebte Feinheitsgrad der Positionsbestimmung eines Elementes im Feld liegt bei einer Differenzierung bis auf die gegenseitige HyponymieRelation bezüglich des vorgegebenen Aspektes. D.h., die Differenzierung endet auf der Ebene der Synonymie innerhalb des Feldes. Die beherrschende und im Gegensatz zu früheren Vorstellungen befreiende Idee bei der Ausformulierung der Differenzierungsmöglichkeiten ist folgende: Mit einer nicht erfolgten Differenzierung über die Dimensionen läßt sich ganz gut leben, solange sich ein Unterschied mithilfe der Sinnrelationen über den Vergleich zu einem dritten Element im Feld nachweisen läßt.

Steht mit der vorgestellten Konzeption nun die Wortfeldtheorie auf einem besseren Fundament als zu ihren Anfängen? Wir wollen im Zusammenhang mit einigen bedenkenswerten Kritikpunkten versuchen, einer Antwort näherzukommen. Zu solchen Kritikpunkten zähle ich 1. Mosaikvorstellung, 2. Grad der Abhängigkeit untereinander, 3. Vollständigkeit, wobei diese Punkte nicht alle voneinander unabhängig sind.

ad 1. Die Mosaikvorstellung sollte zunächst wohl nichts weiter als ein griffiges Bild für die Strukturiertheit eines Feldes liefern. Trier verwendet zwar das Adjektiv „mosaikartig” zu Anfang seiner Monographie zum Sinnbezirk des Verstandes, er hat dieses Bild aber selbst wohl nie allzu ernst genommen. Zum einen mußte er bereits in seiner praktischen Arbeit Abstriche machen, zum andern klingt er in einigen anderen theoretischen 
Äußerungen viel vorsichtiger. So finden wir in einem Vortrag von 1934 (Trier 1934, S. 157):

„(...) die Außengrenzen des Feldes offenbar recht ungewiß sind, die Zahl der Bestandteile unordentlicherweise zu- und abnehmen kann, und die Binnengrenzen weit davon entfernt, als klare mathematische Grenzkonturen sich zu erweisen, in Wahrheit vielmehr Überschneidungszonen und schwankende Übergangsräume darstellen (...)."

Diese Aussage mutet geradezu chaotisch an und könnte von den Kritikern der Wortfeldtheorie nicht schärfer formuliert werden. Ich selbst würde keineswegs so extrem ins 'unsystematische' Lager umschwenken, sondern zum einen immerhin die von uns ermittelte Strukturiertheit gelten lassen wollen. Zum anderen wird der Hinweis auf Fakten der Sprache ganz zu Anfang meiner Ausführungen an dieser Stelle einschlägig: Trier nimmt mit diesem Zitat Einsichten der kognitiven Psychologie und kognitiven Linguistik teilweise vorweg. Es handelt sich bei den von ihm angeführten Erscheinungen um Fakten der Kategorisierung. Insofern dürfen solche Erscheinungen nicht als Schwachpunkte der Wortfeldkonzeption bezeichnet werden.

ad 2. Die Abhängigkeit der Elemente eines Feldes untereinander geht bei meiner Konzeption soweit, daß wir sagen können, aufgrund des gemeinsamen Aspektes sind die Elemente alle in einem globaleren Sinne ähnlich zueinander und sie stehen ferner über die Sinnrelationen in engeren Beziehungen zueinander. Da die Struktur der Inhaltsseite jedoch keine (Gesamt)Lesarten oder gar (Gesamt)Bedeutungen konstituiert, ist nicht ausgeschlossen, daß ein Element sowohl durch die Mitgliedschaft in anderen Wortfeldern als auch durch übergreifendere Beziehungen im gesamten Wortschatz weitere Inhaltsanteile gewinnt. Zu letzteren gehören insbesondere auch Beziehungen zwischen Elementen über Wortartgrenzen bzw. Grenzen syntaktischer Kategorien hinweg, etabliert etwa durch spezielle Kollokationen oder Assoziationen. Es ist für mich auch gar keine Frage, daß einige Elemente des Wortschatzes durch die direkte Erfahrung von Referenzakten eingeführt werden und mit diesem Bezug auch ziemlich stabil verbunden bleiben. Dies gilt sicherlich für einige Elemente im Feld der Verwandtschaftsbezeichnungen, z.B. für mutter. Allerdings sind dies keine typischen Fälle im Wortschatz, da wir hier gleichzeitig wissen, $\mathrm{da} B$ es sich um genau eine Person handelt. Sobald wir Elemente betrachten, deren Extension vielfältige Mitglieder aufweisen, sollte einleuchten, daß die Präzision der Vorstellung sehr wohl vom Auftreten weiterer ähnlicher Elemente abhängt. Man denke an den Besitz des Substantivs baum allein vs. an den Besitz der Substantive baum, busch und blume. Obwohl die Grenzen zwischen dem Inhalt von baum und dem Inhalt von busch niemals eindeutig sein werden, erzwingt allein das Wissen über die Ver- 
schiedenheit wenigstens eines der Stereotype von baum als Gewächs von wenigstens einem der Stereotype von busch als Gewächs zu einer Abgrenzung in den Inhalten der Substantive.

ad 3. 'Vollständigkeit' ist in Form seiner Operationalisierung als Abgeschlossenheit gegenüber den Sinnrelationen für uns kein problematisches Thema mehr. Bei Fällen, die auf den Gesamtrahmen bezogen weiterhin abzuwägen sind, kommt schlieBlich die Intuition zum Zuge. Auch dagegen ist meiner Meinung nach nichts einzuwenden.

Insgesamt haben wir mit der vorgestellten Konzeption die Kritik an den traditionellen Vorstellungen ernst genommen und somit kann man wohl mit Fug und Recht sagen, daß diese Wortfeldkonzeption auf einem besseren Fundament steht.

\section{Was könnten Wortfelder bzw. lexikalische Felder sein?}

Da die Konzeption selbst offensichtlich fundiert ist, muß es bei der Beantwortung dieser Frage eher um die Suche nach geeigneten Anwendungsgebieten der Konzeption gehen. Ich möchte hierzu ganz kurz einige Hinweise geben.

1. Ureigentliches Anwendungsgebiet ist und bleibt selbstverständlich die feldmäBige Behandlung von Ausschnitten des Wortschatzes. Die mit der Konzeption verbundene Rahmenbildung liefert dabei einen willkommenen Fixpunkt für die Erfassung intrasprachlicher und intersprachlicher Vielfalt der Lexikologie. Typologische und vergleichende Untersuchungen der theoretischen Linguistik und sprachdidaktische Übungen der angewandten Linguistik könnten von einem stärkeren Bewußtsein hierüber und einer reichhaltigeren Datenbasis profitieren. Nur über den verstärkten Einsatz computergestützter Verfahren wird eine Datenbasis im wünschenswerten Umfang zu erzielen sein. ${ }^{18}$

2. Eine entsprechende Datenbasis wäre für die Computerlinguistik von unschätzbarem Wert, könnte sie doch für maschinelle Übersetzungsprogramme Anwendung finden.

3. Eine entsprechende Datenbasis müßte schließlich auch für die Praxis der Lexikographie Anlaß genug sein, die Ergebnisse der Lexikologie besser umzusetzen. Neue Formate von Wörterbüchern in Abkehr von der alphabetischen Ordnung sind hier gefragt, auf die der Benutzer/die

18 Auch Bergmann (1989) dringt bei seiner Untersuchung nicht zu einer Wortfeldanalyse vor. 
Benützerin eigentlich schon länger ein Recht hat, dieses Recht ihm/ihr aber aus verschiedenen Gründen verwehrt wird. ${ }^{19}$

4. Wie bereits angedeutet, betrachte ich die Wortfeldtheorie als den eigentlichen Vorläufer der kognitiven Linguistik. Die praktische Arbeit hat hier im Grunde schon immer die von den kognitiven Wissenschaften später ermittelten Erkenntnisse vorweggenommen. Nur wurde dies durch die über lange Zeit anscheinend untrennbare Verbindung zwischen Wortfeldanalyse und einer an klassischen, aristotelischen Vorstellungen orientierten Merkmalsbeschreibung zugedeckt. Erst mit der Befreiung von dieser Verbindung in den $80 \mathrm{er}$ Jahren wurde die Relevanz der Wortfeldtheorie für den kognitiven Ansatz offensichtlich. Dabei kann die kognitive Linguistik durchaus noch hilfreiche Anstöße von der Wortfeldkonzeption erwarten. Zwei Bereiche habe ich hiervon in letzter Zeit angedeutet:

a) Im Bereich der Kategorisierung ist neben der Erfassung der Struktur im Innern einer Kategorie auch die Erfassung der Art und Weise der Grenzziehung nach außen zu anderen Kategorien zumindest genauso wichtig. Dafür bietet sich die Berücksichtigung des Feldansatzes geradezu an. ${ }^{20}$ Entsprechende Vorteile bei der mehr oder weniger analogen Aufstellung künstlicher Interpretationen im Stile der Modelltheorie wurden von mir schon länger hervorgehoben. ${ }^{21}$

b) Im Bereich der Schnittstelle 'Syntax/Semantik' läßt sich eine vernünftigere Beschreibung bei Berücksichtigung der in Feldern vorliegenden Informationen erzielen. Ich denke hier in erster Linie an die Anforderungen an eine syntaktische Analyse, die im Sinne einer Arbeitsteilung von manchen Angaben entlastet werden könnte. ${ }^{22}$ Umgekehrt kann selbstverständlich auch die Wortfeldtheorie durch Begrifflichkeiten und Methoden der kognitiven Linguistik bereichert werden. Je mehr dies geschehen wird, um so mehr wird auch die Verbindung zwischen Wortfeldtheorie und klassischen strukturellen Verfahren gelockert werden.

Für die Überlebenschancen der Wortfeldtheorie muß es einem danach wahrlich nicht bange sein. Die die Inhaltsseite betreffenden Vorstellungen der Wortfeldtheorie sind ja allenfalls der natürlichen Semantik verpflich-

19 Schumacher (1986) kann hier als interessanter Versuch gewertet werden.

20 Mehr Informationen hierzu finden sich in Lutzeier (1993b).

21 Vgl. Lutzeier (1983a).

22 Vgl. Lutzeier (1992). 
tet, dafür jedoch unabhängig von jeglicher künstlicher (lexikalischer) Semantik. ${ }^{23}$

5. Zwischen Psychologie und Linguistik wäre ein besserer Austausch über die jeweiligen Feldvorstellungen wünschenswert. ${ }^{24}$ Ziel hierbei müßte letztlich sein, herauszufinden, in welchem AusmaB und bei welchen Aufgabenstellungen wortfeldartige Gruppierungen im mentalen Lexikon eine Rolle spielen. Wie zu Anfang gesagt, bin ich davon überzeugt, daß unsere Felder psychologische Realität besitzen.

6. Die auf Weinrich zurückgehende Bildfeldkonzeption könnte in diesem Zusammenhang eventuell einen völlig neuen Anwendungsbereich finden. Ich denke an die inzwischen von Peil (1993) versuchten Präzisierungen, die nicht nur die syntagmatische Orientierung hervorheben, sondern auch mit den Beispielen eine Ablösung von der rein sprachlichen Ebene suggerieren. Unter der Annahme, daB nun auf einigen Verarbeitungsebenen gerade nicht-sprachliche Repräsentationen eine Rolle spielen, müßte auch eine solche Feldkonzeption für die Untersuchung des mentalen Lexikons Berücksichtigung finden.

Kormmen wir zum Schluß: Lexikalische Felder sind sowohl Zeugen als auch Kristallisationspunkt für insgesamt in einer natürlichen Sprache wirkende feldmäBige Kräfte. Die Linguistik hat somit die Aufgabe, dieser Tatsache in ihrer Theoriebildung und praktischen Arbeit gerecht zu werden.

\section{Literatur}

Abel, Carl (1878): Die englischen Verba des Befehls. Berlin: Verlag von Leo Liepmannssohn.

Abel, Carl (1882): On philological methods. In: C. Abel: Linguistic Essays. London: Trübner \& Co. S. 157-167.

Becker, Hans-Joachim (1991): Das Feld um alt. Heidelberg: Carl Winter Universitätsverlag.

Bergmann, Christian (1989): Zur Anwendung quantitativer und qualitativer Ermittlungsverfahren bei textgebundenen Wortfelduntersuchungen. In: Zeitschrift für Germanistik 10, S. 589-596.

Coseriu, Eugenio (1967): Lexikalische Solidaritäten. In: Poetica 1, S. 293-303.

23 Für das Begriffspaar 'natürliche Semantik' und 'künstliche Semantik' vgl. Lutzeier (1985a, S. 59ff.).

24 Tischer (1988) liefert einen exzellenten Ūberblick über die in der Psychologie vorherrschenden Verfahren. 
Coseriu, Eugenio (1970): Einführung in die strukturelle Betrachtung des Wortschatzes. In: E. Coseriu: Einfūhrung in die strukturelle Betrachtung des Wortschatzes. Tübingen: Gunter Narr (2. Auflage 1973). S. 1-104.

Coserin, Eugenio (1979): Zur Vorgeschichte der strukturellen Semantik: Heyses Analyse des Wortfeldes 'Schall'. In: E. Coseriu: Sprache. Strukturen und Funktionen. Tübingen: Gunter Narr Verlag (3. Auflage). S. 149-159.

Frege, Gottlob (1892): Ūber Sinn und Bedeutung. In: Zeitschrift für Philosophie und philosophische Kritik 100, S. 25-50. Abgedruckt in: Gottlob Frege: Kleine Schriften (Hg. I. Angelelli). Darmstadt: Wissenschaftliche Buchgesellschaft 1967 (2.Auflage). S. 143-162.

Geckeler, Horst (1971): Strukturelle Semantik und Wortfeldtheorie. Mūnchen: Fink Verlag.

Heyse, Karl Wilhelm Ludwig (1856): System der Sprachwissenschaft (Hg. v. H. Steinthal). Berlin.

Ipsen, Gunther (1924): Der alte Orient und die Indogermanen. In: Stand und Aufgaben der Sprachwissenschaft. Festschrift fūr W. Streitberg (Hg. J. Friedrich et al.). Heidelberg: Carl Winter's Universitätsbuch handlung. S. 200-237.

Jolles, André (1934): Antike Bedeutungsfelder. In: Beiträge zur Geschichte der deutschen Sprache und Literatur 58, S. 97-109. Abgedruckt in: Schmidt (1973), S. 104-115.

Lipka, Leonard (1980): Methodology and representation in the study of lexical fields. In: Perspektiven der lexikalischen Semantik. Beiträge zum Wuppertaler Semantikkolloquium vom 2.-3. Dezember 1977 (Hg. D. Kastovsky). Bonn: Bouvier. S. 93-114.

Lutzeier, Peter Rolf (1981): Wort und Feld. Wortsemantische Fragestellungen mit besonderer Berücksichtigung des Wortfeldbegriffes. Tübingen: Max Niemeyer Verlag.

Lutzeier, Peter Rolf (1983a): Wortfelder als Maßstab für Interpretationen am Beispiel des Feldes der Stimmungen im Deutschen. In: Zeitschrift für Sprachwissenschaft 2, S. 45-71.

Lutzeier, Peter Rolf (1983b): The relevance of semantic relations between words for the notion of lexical field. In: Theoretical Linguistics $10(2 / 3)$, S. 147-178.

Lutzeier, Peter Rolf (1985a): Linguistische Semantik. Stuttgart: Metzlersche Verlagsbuchhandlung.

Lutzeier, Peter Rolf (1985b): Die semantische Struktur des Lexikons. In: Handbuch der Lexikologie (Hg. C. Schwarze/D.Wunderlich). Königstein/Ts.: Athenäum-Verlag. S. 103-133.

Lutzeier, Peter Rolf (1992): Wortfeldtheorie und kognitive Linguistik. In: Deutsche Sprache 20, S. 62-81.

Lutzeier, Peter Rolf (1993a) (Hg.): Studien zur Wortfeldtheorie/Studies in Lexical Field Theory. Tübingen: Max Niemeyer Verlag.

Lutzeier, Peter Rolf (1993b): Wortfelder als kognitive Orientierungspunkte? In: Lutzeier (1993a), S. 203-214. 
Lutzeier, Peter Rolf (1993c): Anmerkungen zur Wortbedeutung. In: Wortschatz - Satz - Text. Beiträge der Konferenzen in Greifswald und Neubrandenburg 1992 (Hg. G.Bartels/I.Pohl). Frankfurt a.M.: Peter Lang. S. 257-268.

Meyer, Richard M. (1910a): Bedeutungssysteme. In: Zeitschrift für vergleichende Sprachforschung 43, S. 352-368.

Meyer, Richard M. (1910b): Die militärischen Titel. In: Zeitschrift für Deutsche Wortforschung 12, S. 145-156.

Paul, Hermann (1992): Deutsches Wörterbuch (9. Auflage, bearbeitet von H.Henne/G.Objartl). Tübingen: Max Niemeyer Verlag.

Peil, Dietmar (1993): Zum Problem des Bildfeldbegriffs. In: Lutzeier (1993a), S. 185-202.

Porzig, Walter (1934): Wesenhafte Bedeutungsbeziehungen. In: Beiträge zur Geschichte der deutschen Sprache und Literatur (Halle) 58, S. 70-97. Abgedruckt in: Schmidt (1973), S. 78-103.

Röhrich, Lutz (1973): Lexikon der sprichwörtlichen Redensarten Band 4: Sitzfleisch bis 2 wölf. Freiburg: Herder (4. Auflage).

Schindler, Wolfgang (1993): Phraseologismen und Wortfeldtheorie. In: Lutzeier (1993a), S. 87-106.

Schlaefer, Michael (1987): Studien zur Ermittlung und Beschreibung des lexikalischen Paradigmas 'lachen' im Deutschen. Heidelberg: Carl Winter Universitătsverlag.

Schmidt, Lothar (1973) (Hg.): Wortfeldforschung. Zur Geschichte und Theorie des sprachlichen Feldes. Darmstadt: Wissenschaftliche Buchgesellschaft.

Schumacher, Helmut (1986) (Hg.): Verben in Feldern. Valenzwörterbuch zur Syntax und Semantik deutscher Verben. Berlin: Walter de Gruyter.

Tischer, Bernd (1988): Kein Spaß im Wortfeld der Gefühlsbegriffe? Zehn Methoden zur Kennzeichnung der Wortfeldzugehörigkeit. In: Archiv für Psychologie 140 (1), S. 15-31.

Trier, Jost (1931): Der deutsche Wortschatz im Sinnbezirk des Verstandes. Die Geschichte eines sprachlichen Feldes. Band 1 (Von den Anfängen bis zum Beginn des 13. Jahrhunderts). Heidelberg: Carl Winter (2. Auflage 1973).

Trier, Jost (1934): Das sprachliche Feld. Eine Auseinandersetzung. In: Neue Jahrbücher für Wissenschaft und Jugendbildung 10, S. 428-449. Abgedruckt in: Schmidt (1973), S. 129-161.

Wander, Karl F.W. (1876): Deutsches Sprichwörter-Lexikon. Ein Hausschatz für das deutsche Volk. Vierter Band: Sattel bis Wei. Leipzig: F.A.Brockhaus.

Weisgerber, Leo (1951/52): Zur innersprachlichen Umgrenzung der Wortfelder (veranstalten und stattfinden). In: Wirkendes Wort 2, S. 138-143. Abgedruckt in: Schmidt (1973), S. 278-287. 